University of Nebraska - Lincoln

DigitalCommons@University of Nebraska - Lincoln

U.S. Department of Veterans Affairs Staff

Publications

U.S. Department of Veterans Affairs

2002

\title{
Comparing attentional bias to smoking cues in current smokers, former smokers, and non-smokers using a dot-probe task
}

\author{
Ronald N. Ehrman \\ University of Pennsylvania, ehrman_r@mail.trc.upenn.edu \\ Steven J. Robbins \\ Arcadia University, robbinss@arcadia.edu \\ Melissa Bromwell \\ University of Pennsylvania \\ Megan E. Lankford \\ Arcadia University \\ John R. Monterosso \\ University of Pennsylvania, johnrmon@usc.edu \\ See next page for additional authors
}

Follow this and additional works at: https://digitalcommons.unl.edu/veterans

\begin{abstract}
Ehrman, Ronald N.; Robbins, Steven J.; Bromwell, Melissa; Lankford, Megan E.; Monterosso, John R.; and O'Brien, Charles P., "Comparing attentional bias to smoking cues in current smokers, former smokers, and non-smokers using a dot-probe task" (2002). U.S. Department of Veterans Affairs Staff Publications. 50. https://digitalcommons.unl.edu/veterans/50
\end{abstract}

This Article is brought to you for free and open access by the U.S. Department of Veterans Affairs at DigitalCommons@University of Nebraska - Lincoln. It has been accepted for inclusion in U.S. Department of Veterans Affairs Staff Publications by an authorized administrator of DigitalCommons@University of Nebraska - Lincoln. 


\section{Authors}

Ronald N. Ehrman, Steven J. Robbins, Melissa Bromwell, Megan E. Lankford, John R. Monterosso, and Charles P. O'Brien 


\title{
Comparing attentional bias to smoking cues in current smokers, former smokers, and non-smokers using a dot-probe task
}

\author{
Ronald N. Ehrman ${ }^{\mathrm{a}, \mathrm{c}, *}$, Steven J. Robbins ${ }^{\mathrm{b}}$, Melissa A. Bromwell ${ }^{\mathrm{a}}$, Megan \\ E. Lankford ${ }^{\mathrm{b}}$, John R. Monterosso ${ }^{\text {a }}$, Charles P. O'Brien ${ }^{\mathrm{a}, \mathrm{c}}$ \\ ${ }^{a}$ Treatment Research Center, University of Pennsylvania, 3900 Chestnut Street, Philadelphia, PA 19104, USA \\ b Department of Psychology, Arcadia University, 450 South Easton Road, Glenside, PA 19038, USA \\ ${ }^{\mathrm{c}}$ Veteran's Affairs Medical Center, 39Th and Woodland Aves, Philadelphia, PA 19104, USA
}

Received 20 August 2001; received in revised form 17 March 2002; accepted 22 March 2002

\begin{abstract}
Much evidence documents that individuals with emotional and drug-use disorders demonstrate biased attention toward stimuli associated with their disorder. This bias appears to diminish following successful treatment. Two studies examined whether current cigarette smokers show biased attention toward smoking-related images compared with non-smokers (Studies 1 and 2) and whether this bias is less pronounced in former smokers (Study 2). Attentional bias toward cigarette-related photographs was examined using the dot-probe task. Pairs of images (one smoking-related) appeared side by side for $500 \mathrm{~ms}$ on a computer screen prior to the presentation of a probe (an asterisk) replacing one of the photographs. Subjects struck a key as quickly as possible to indicate the probe location. Attentional bias was defined as faster reaction times when the probe replaced the smoking-related image. In both studies, current smokers displayed significantly greater attentional bias toward cigarette stimuli than did non-smokers. Former smokers in Study 2 displayed an intermediate level of bias, but did not differ significantly in bias score from either of the other groups. These results support further use of the dot-probe task as a measure of attentional bias in non-abstinent smokers and in individuals undergoing smoking cessation treatment. (C) 2002 Elsevier Science Ireland Ltd. All rights reserved.
\end{abstract}

Keywords: Cigarette smoking; Attention; Dot-probe task; Reaction time

\section{Introduction}

A great deal of research now documents that events related to drug use come to evoke drug-related responses such as high, withdrawal, craving, and arousal (see Robbins and Ehrman, 1992; Drummond et al., 1995; O’Brien et al., 1998 for recent reviews). Many theorists view drug-related cues as a causal factor in drug use and relapse to drug use following treatment (Wikler, 1965; Siegel, 1979; Stewart et al., 1984; Childress et al., 1986; Baker et al., 1987; Tiffany, 1990). In the specific case of smoking, cigarette-related cues have been shown to both increase craving and decrease the latency to smoke (e.g. Abrams et al., 1988; Niaura et al., 1988, 1992; Droungas et al., 1995). Although most

\footnotetext{
* Corresponding author. Tel.: +1-215-222-3200x123; fax: +1-215386-6770

E-mail address: ehrman_r@mail.trc.upenn.edu (R.N. Ehrman).
}

discussions of cue reactivity have not explicitly addressed the role of attention to drug cues, the incentive-sensitization model of Robinson and Berridge (1993) postulates that drug-related stimuli become more salient or attention-grabbing as a consequence of chronic drug use. Such a process could produce a positive feedback loop that magnifies the risk posed by drug-related cues. That is, enhanced attention to drugrelated cues should produce enhanced responding to those cues. Increases in cue-induced responses may promote greater drug use that in turn increases drug cue salience.

Although relatively little effort has been devoted thus far to investigating attentional processes in substance abuse, many studies have examined the role of attention in other emotional disorders. Much of this research has made use of the emotional Stroop color-naming task. In the Stroop task, individuals are asked to call out as quickly as possible the color of the ink in which target 
words appear. Greater interference with color naming (increased verbal response times) is taken as evidence for enhanced attention to the meaning of the target words. In two extensive literature reviews, Williams et al. (1996, 1997) discuss studies demonstrating that individuals with a variety of emotional disorders including depression, generalized anxiety disorder, panic disorder, phobias, and PTSD show increased color-naming times when the target words are disorder-relevant. Two findings in this literature are particularly striking. First, color-naming interference is highly selective; individuals show impairments only for words specifically related to their disorder (e.g. McNally et al., 1990; Watts et al., 1986). Second, successful treatment reduces color-naming interference (Watts et al., 1986; Gotlib and Crane, 1987; Mattia et al., 1993; Mathews et al., 1995). Thus, Stroop interference by affect-laden words appears to be a specific index of an individual's current level of preoccupation or distress.

Several studies in recent years have extended the emotional Stroop task to studies of smokers. Gross et al. (1993) found that active smokers who are asked to abstain from smoking for $12 \mathrm{~h}$ before a test session show greater Stroop interference with cigarette-related words than with control words. Similar results were reported by Waters and Feyerabend (2000) using smokers who were deprived of cigarettes for $24 \mathrm{~h}$ prior to testing. However, these studies did not find interference differences between smoking-related words and control words in non-abstinent smokers and did not test non-smokers. A third study (Johnson et al. 1997) compared current smokers, recent quitters, and non-smokers and failed to find an overall effect of group on Stroop performance. Most recently, Wertz and Sayette (2001) examined Stroop performance in smokers who were told that they would or would not be allowed to smoke during the experiment. Individuals given an expectation of smoking showed a greater degree of color-naming interference with smoking words. However, all subjects underwent testing following $12 \mathrm{~h}$ of smoking deprivation. Thus, attentional bias effects in non-abstinent smokers remain to be demonstrated in the Stroop task.

A second test used to study attentional bias is the dotprobe task first introduced by MacLeod et al. (1986). Subjects are shown two images (pictures or words) sideby-side on a computer screen. When the two images disappear, a target stimulus (the dot probe) appears in place of one of the images. Subjects are asked to indicate the location of the target by striking a key as quickly and accurately as possible. Faster reaction times (RTs) when the target replaces a particular class of events indicate biased attention toward those events. The dotprobe task has been used successfully to demonstrate that individuals with mood disorders such as anxiety or depression shift attention toward events related to those states (MacLeod et al., 1986; MacLeod and Mathews,
1988; Mogg et al., 1992, 1994, 1995, 1997). Induced mood states can have similar effects; hungry individuals (who do not suffer from an eating disorder) show bias toward food-related stimuli (Mogg et al. 1998). Finally, and of most interest here, selective attention effects have also been shown in users of opiates (Lubman et al., 2000) and alcohol (Townshend and Duka, 2001) when presented with pictures of drug-related stimuli in the dot-probe task. The present study was designed to extend the use of the dot-probe task to the study of smokers.

Because cue-induced craving is thought to play an important role in smoking, biased visual attention toward cigarette-related cues may increase the motivation to smoke. Smokers may be more likely than nonsmokers to notice in passing an ashtray or pack of cigarettes, thereby increasing their likelihood of experiencing cue-induced craving. The dot-probe task appears better designed than the Stroop task to study such shifts in the direction of visual attention for several reasons.

First, while the Stroop task requires subjects to attend to a single target word, the dot-probe task explicitly examines shifts in attention between two co-present visual events. In this way, the dot-probe task better simulates real-world conditions in which both smokingrelated and non-smoking-related objects compete for visual attention. Second, color-naming interference on the Stroop task could result from processes other than initial shifts in attention. For example, words associated with an individual's area of concern might produce greater interference than control words because they produce cognitive/mood states which compete with the color-naming response (e.g. Williams et al., 1996). By contrast, the dot-probe task measures biased attention as the facilitation of target location performance when the target and smoking picture appear in the same position. Targets that appear in place of smoking pictures cannot be responded to faster unless attention has already been directed toward those pictures. Finally, Stroop studies have only produced interference effects in smokers who abstained from smoking prior to the test session. We hoped that the dot-probe task would prove to be a more sensitive measure of biased attention in non-abstinent smokers.

The two studies reported here compare attentional bias to cigarette-related photographs across groups with different smoking histories. Study 1 represented a small pilot study examining whether current smokers show greater attentional bias to smoking-related photographs than non-smokers. Study 2 represented a replication of Study 1 with larger and more diverse samples and the inclusion of a group with a prior smoking history (former smokers). As noted earlier, several Stroop task studies have demonstrated that individuals with emotional disorders show reduced Stroop interference by disorder-related words following successful treatment 
(Watts et al., 1986; Gotlib and Crane, 1987; Mattia et al., 1993; Mathews et al., 1995). By analogy, we predicted that former smokers would show less bias toward smoking-related events than current smokers.

\section{Methods}

\subsection{Subjects}

A total of seven smokers (six White and one Asian) and 23 non-smokers (17 White, four Black, two Asian) participated in Study 1. Non-smokers (eight males, 15 females) had a mean age of 34.5 (S.D. $=11.3$ ) and reported having never smoked cigarettes regularly (a few reported smoking a single cigarette on isolated occasions). Smokers (two males, five females) had a mean age of 27.1 (S.D. $=6.4$ ) and reported smoking an average of 7.3 (S.D. $=6.1$ ) cigarettes per day. Individuals in this study were a convenience sample of staff at our Treatment Research Center and received no reimbursement for their participation. The study was IRBapproved and all participants gave informed, written consent at the outset.

Three groups of individuals took part in Study 2: current smokers $(n=67)$, non-smokers $(n=25)$, and former smokers $(n=16)$. Current smokers (29 White, 34 Black, two Asian, one Hispanic, one Unknown) consisted of 30 males and 37 females with a mean age of 36.7 (S.D. $=12.8$ ). They reported smoking an average of 20 cigarettes per day (S.D. $=13.4)$. Thus, the smokers in this study smoked considerably more heavily than those in Study 1. Non-smokers (18 White, six Black, one Hispanic) consisted of six males and 19 females with a mean age of 23.1 (S.D. $=2.7$ ). They reported never having regularly smoked cigarettes. Former smokers consisted of individuals who self-reported having quit smoking for at least 1 week. This group (13 White, two Asian, one Hispanic) contained six males and ten females. Their mean age was 37.1 (S.D. $=12.7)$ and they reported having last smoked an average of 6.5 years (S.D. $=8.8$, range $=10$ days to 30 years) prior to the study.

Most of the individuals in Study 2 were recruited through referral from a smoking cessation program at the Treatment Research Center and through word-ofmouth. These subjects received $\$ 20$ for their participation in the session. A total of six non-smokers and 12 current smokers were undergraduate students at a local college who participated in the on-campus study as part of a course requirement. These subjects were not paid for their participation but were entered into a lottery for a $\$ 35$ gift certificate. All subjects gave written, informed consent to participate and the study was IRB-approved at both locations.

\subsection{Apparatus and stimuli}

The apparatus and stimuli were identical in the two studies. The dot-probe task was programmed and presented using E-Prime version 1.0 (Psychology Software Tools, Inc.). The program was run on a Windows $98 \mathrm{PC}$ and stimuli were presented on a 17 -in. monitor. The pictures used in the dot-probe task were taken by study personnel using a digital camera and organized into matched pairs of photographs. Individual images were identically sized to $11 \mathrm{~cm}$ wide by $13.5 \mathrm{~cm}$ high using Adobe Photo Shop and were presented $10 \mathrm{~cm}$ apart. Each pair contained one picture with content related to cigarette smoking (pack of cigarettes, a hand holding a cigarette) and a matched picture with content not specifically related to cigarettes (pack of playing cards, a hand holding a pen). Photographs were matched for overall composition and degree of visual complexity.

Thirty picture pairs were used in the study. Ten of the pairs contained two photographs without smoking content. These photographs were used during initial practice trials. Twenty pairs of photographs contained one picture with smoking content.

Response latencies were collected by means of a response box containing two buttons labeled L (left) and $\mathrm{R}$ (right). The box was interfaced with the computer running E-Prime. The computer recorded response latencies to the nearest tenth of a millisecond.

\subsection{Procedure}

Except where indicated, the two studies employed identical procedures similar to those used by Lubman et al. (2000) and by Townshend and Duka (2001). Subjects reported to the laboratory for a single test session. After giving written consent, subjects were seated $1 \mathrm{~m}$ in front of the computer screen and given a verbal description of the task. They were told that pairs of pictures would briefly flash on the screen followed by an asterisk (the dot probe) in the position formerly occupied by one of the pictures. Subjects were instructed to strike the left or right response key as quickly and accurately as possible to indicate the position of the target.

Following the instructions, the subjects received 20 practice trials employing ten pairs of photographs presented twice each. The photograph pairs contained no pictures with smoking-related content and were not used during the main body of the task. A rest period followed that terminated when the subject pressed a computer key. Subjects then received 80 trials without a break. Each trial began with the presentation of a fixation point $(\mathrm{X})$ in the center of the computer screen for $1 \mathrm{~s}$. Immediately following offset of the fixation point, a pair of photographs was presented for $500 \mathrm{~ms}$. In each pair, one of the pictures appeared to the left of 
the fixation point and one appeared to the right. When the images disappeared, an asterisk immediately appeared in the place of one of the images. The target remained on the screen until the subject struck a response key or for a total of $2 \mathrm{~s}$ if no key was struck.

Each of the picture pairs was presented four times during the study: twice with the smoking picture appearing on the left and twice with the smoking picture on the right. For each configuration, the target stimulus replaced the smoking picture on one occasion and replaced the non-smoking picture on the other occasion. Thus, there were four trial types created for each stimulus pair: (1) smoking picture and target both on left, (2) smoking picture and target both on right, (3) smoking picture on left, target on right, and (4) smoking picture on right, target on left.

Stimuli were presented in two blocks of 40 trials each. In each block, one configuration of each stimulus pair (e.g. smoking picture on left) was presented twice, once with the target replacing the smoking picture and once with the target replacing the non-smoking stimulus. In this way, half the trials in each block presented the target on the same side as the smoking picture. Similarly, half the picture pairs in each block presented the smoking picture on the left side and half presented this picture on the right. The two blocks were presented in a fixed order to all participants. Trial order within a block was randomly determined for each individual by E-Prime.

The procedure for Study 2 differed from Study 1 only in the inclusion of several self-report scales before and after the dot-probe task. Subjects were asked to fill out the Shiffman-Jarvik nicotine withdrawal schedule (Shiffman and Jarvik, 1976), the Profile of Mood States (POMS) questionnaire (McNair et al., 1971), and two visual analog scales (VAS) asking them to rate their current levels of craving and withdrawal on a $100 \mathrm{~mm}$. line. Scales were anchored with the words 'Not at All' and 'Most Ever' at the two ends. The 18 undergraduate students in the study received only the two VAS forms before and after the task. With the exception of the VAS forms given to the undergraduates, all self-report data were collected through computerized test forms presented to subjects on a laptop PC.

\subsection{Data reduction and analysis}

Trials with errors were not used in mean reaction time calculations. In addition, trials with reaction times of less than $200 \mathrm{~ms}$ or greater than $1000 \mathrm{~ms}$ were eliminated from the analyses (Townshend and Duka, 2001). Values less than $200 \mathrm{~ms}$ indicate that the subject initiated a response before the onset of the target. Reaction times greater than $1000 \mathrm{~ms}$ suggest anomalous processing during the trial such as inattention to the task or motor error (e.g. finger slipping). Less than $3 \%$ of trials were eliminated from the analyses in both studies and no group differences in data elimination were observed.

For each subject, mean RTs were calculated for trials when the target replaced the smoking photograph and for trials when the target replaced the non-smoking photograph. Attentional bias was then expressed through a difference score calculated as non-smoking RT-smoking RT (Lubman et al., 2000). Thus, positive bias scores reflect faster RTs when the target replaces the smoking pictures. In Study 1, an independent-groups $t$-test was employed to compare the bias scores of the two groups (current smokers and non-smokers). In Study 2, a one-way ANOVA was used to compare bias scores across the three groups (current smokers, former smokers, non-smokers). Direct comparisons of group bias scores are statistically equivalent to examining Group X Stimulus Type interactions in a 2-way repeated-measures ANOVA using raw RTs. Tukey's HSD was used to make post-hoc comparisons between pairs of groups. Alpha was set at 0.05 for all analyses.

\section{Results}

\subsection{Results of Study 1}

Mean reaction times for the two groups are depicted in Table 1. Although both groups were somewhat faster to respond to the target when it replaced the smoking stimulus, this bias was more than three times greater in the smokers. Results of the $t$-test demonstrated that this group difference in bias scores was significant $(t(28)=$ $2.48, P<0.05)$.

\subsection{Results of Study 2}

\subsubsection{Reaction times}

Mean reaction times for the three groups are shown in Table 2. The three groups were ordered as predicted; non-smokers showed the least bias and current smokers showed the greatest bias. The one-way ANOVA revealed a significant group effect, $F(2,105)=3.24, P<$ 0.05). Post-hoc comparisons using Tukey's HSD found that the bias scores for the non-smokers and current smokers differed significantly from one another $(P<$ 0.05). However, the mean bias score for the former smokers was not significantly different from the mean for either of the two other groups $(P \mathrm{~s}>0.05)$.

\subsubsection{Self-reports}

In Study 2, we attempted to collect self-report data by having subjects respond to computer-based questionnaire items. Unfortunately, both software problems and participant difficulties in properly entering responses resulted in considerable loss of self-report data. Because the non-smoker and former smoker groups had small 
Table 1

Mean (S.E.M.) reaction times (ms) in Study 1 comparing attentional bias in non-smokers and current smokers

\begin{tabular}{lllr}
\hline & Target replaces control picture & Target replaces smoking picture & Bias score \\
\hline Non-smokers $(n=23)$ & $468.1(21.5)$ & $461.6(21.2)$ & $6.5(2.3)$ \\
Current smokers $(n=7)$ & $426.2(30.1)$ & $403.2(26.4)$ & $23.0(9.8)$ \\
\hline
\end{tabular}

Reaction times reflect the latency to press a response key indicating the location of a target (asterisk) which replaced either a smoking or matched non-smoking (control) picture on a screen. Bias scores are calculated by subtracting the smoking picture score from the control picture score. Positive scores indicate biased attention toward smoking pictures.

sample sizes to begin with, between-group comparisons of self-report scores were not feasible. However, sufficient data were collected from current smokers to permit Pearson correlations to be calculated between self-report scores and reaction time bias scores. Such correlations were calculated for pre-test measures of craving and withdrawal on the VAS, for each of the six mood scales on the POMS (depression, anger, tension, confusion, fatigue, and vigor), and for the overall negative mood score produced by the POMS. Each of these correlations employed at least 50 of the 67 subjects in the current smoker group. All nine correlations produced values of $r<0.10$ and none approached statistical significance at the 0.05 level. Thus, there was no evidence that bias scores in the current smoker group varied as a function of general mood states, craving, or withdrawal present at the time of testing.

\section{Discussion}

The results of both studies demonstrate that smokers show biased attention toward smoking-related stimuli. This bias did not result from the intrinsic salience or visual attractiveness of the smoking stimuli; non-smoking subjects showed significantly less bias toward the smoking pictures. Former smokers showed an intermediate level of bias. However, their bias scores did not significantly differ from either the current smokers or non-smokers on pairwise comparisons.

The consistency of this attentional bias result across two studies is particularly striking given the large variations in overall reaction times across groups. In Study 1, current smokers had overall reaction times that were 40-60 ms faster than non-smokers. In Study 2, that difference was reversed; current smokers had considerably longer reaction times than both former smokers and nonsmokers. These variations were due to our use of convenience samples across the two studies; our participants varied considerably in demographic characteristics which may have influenced overall reaction times on the task. Most strikingly, current smokers were much younger than non-smokers in Study 1. In Study 2, this age difference was reversed. Thus, overall RT differences may simply have been age-related. However, despite these variations across groups, the within-subject bias pattern persisted. In both studies, biased attention to smoking pictures was significantly greater in current smokers than in comparison groups. Thus, current smokers showed the greatest tendency to attend to smoking pictures regardless of whether their overall reactions times were relatively fast or relatively slow.

These results extend the results of Stroop task studies of attentional bias in smokers in three ways. First, the present findings demonstrate differences in attentional bias between cigarette smokers and individuals with no past history of smoking. Second, biased attention toward smoking scenes was demonstrated without requiring subjects to abstain from smoking prior to the dot-probe task. Because we did not intend to study the effects of deprivation, smokers were free to smoke according to their usual pattern right up to the time of testing. This approach has the disadvantage of allowing variables such as time since last cigarette to vary across participants. However, this tactic also increases the external validity of our findings by demonstrating that attentional bias effects could be detected under naturalistic smoking conditions. Finally, the findings of Study

Table 2

Mean (S.E.M.) reaction times (ms) in Study 2 comparing attentional bias in non-smokers, former smokers and current smokers

\begin{tabular}{lllr}
\hline & Target replaces control picture & Target replaces smoking picture & Bias score \\
\hline Non-smokers $(n=25)$ & $423.2(15.1)$ & $421.9(15.7)$ & $1.3(3.5)$ \\
Former smokers $(n=16)$ & $393.5(13.9)$ & $384.7(13.6)$ & $8.8(4.5)$ \\
Current smokers $(n=67)$ & $467.5(12.6)$ & $455.7(12.4)$ & $11.8(2.1)$ \\
\hline
\end{tabular}

Reaction times reflect the latency to press a response key indicating the location of a target (asterisk) which replaced either a smoking or matched non-smoking (control) picture on a screen. Bias scores are calculated by subtracting the smoking picture score from the control picture score. Positive scores indicate biased attention toward smoking pictures. 
2 are consistent with the idea that attentional bias in smokers diminishes as a result of long-term abstinence.

The results of this study are consistent with findings from other tasks suggesting that smoking-related events capture the attention of smokers. A number of studies have examined whether induced craving for smoking interferes with concurrent tasks. For example, CepedaBenito and Tiffany (1996) asked current smokers to imagine scenes which were either urge-related or not. At the same time, subjects participated in a tone detection task. Urge imagery interfered with tone detection more greatly than non-urge imagery, suggesting that imagined smoking scenes made greater use of attentional resources. Sayette and Hufford (1994) similarly found that performance on an auditory detection task was more greatly impaired by exposure to smoking-related objects than by exposure to control objects. These results could reflect differences in mood states evoked by the different images/cues or differences in the complexity of the imagined scenes or tasks. However, they are also consistent with the idea that smokingrelated events capture the attentional resources of smokers.

Several limitations of the present results are worth noting. First, because of constraints imposed by a timelimited undergraduate project, the student participants in Study 2 were not administered the same self-report battery as the rest of the participants. Thus, bias scores in the undergraduate subgroup could have been influenced by this procedural variation. However, analysis of the subgroup data revealed that these individuals showed a bias pattern across groups (greater bias in smokers than in nonsmokers) similar to that displayed by the rest of the participants. Second, although the mean bias score for the former smokers was intermediate to the scores for current smokers and non-smokers, neither pair-wise comparison was statistically significant. This result could reflect the small sample of former smokers studied. However, it might also reflect the fact that some of the former smokers had quit only days or weeks before the start of the study. Future studies need to examine the relationship between length of abstinence and levels of attentional bias with a larger sample of former smokers.

Third, even if a significant difference in bias score had been found between current and former smokers, that result would not demonstrate that successful smoking cessation was the cause of reduced attentional bias. The natural groups design utilized here leaves open the possibility that the two groups differed in bias scores for other reasons. Only a randomized trial collecting bias scores before and after treatment can conclusively demonstrate that reductions in attentional bias occur when individuals stop smoking.

The fact that the groups used here were convenience samples not matched for demographic or self-report characteristics raises the more general question of whether our results reflect differences in smoking status rather than differences in states such as depression or withdrawal. Although group comparisons of self-reported mood states were not conducted because of the large quantities of missing self-report data, the small samples employed and the lack of explicit matching makes it likely that group differences would have been observed on some measures. However, the correlations conducted using the current smokers in Study 2 provide some evidence that our results reflect smoking status and not the effects of other related variables. Correlations between self-reported mood states (including craving and withdrawal) and reaction time bias scores were near zero, suggesting that bias scores were a reflection of smoking history, not current affective states. Furthermore, the large existing literature on attentional bias effects consistently supports the idea that such effects are specific to an individual's area of concern (e.g. Williams et al., 1996, 1997). In other words, greater depression in the current smokers would be expected to produce greater attentional bias toward depression-related events, not smoking images. Nevertheless, future studies need to replicate our results with better-matched subject samples.

At a more speculative level, the present results suggest several avenues for future studies of attentional bias in smokers. First, the extent of the bias may serve as an initial predictor of treatment outcome. Individuals with more pronounced bias at treatment intake might prove to be less likely to achieve abstinence and/or more likely to relapse following treatment. Second, attentional bias may have utility as an assessment of treatment success. Individuals with the least risk for relapse may be those who show the greatest reductions in bias or who show post-treatment bias scores below a given threshold. Finally, the dot-probe task could be used to examine the conditions that most greatly motivate smoking for a given individual. Sets of smoking scenes could be created which emphasize smoking under different conditions: alone or in groups, when sad or happy, stressed or celebrating, and so on. The specificity of stimulus effects in the literature on emotional disorders encourages the notion that smokers might show greater levels of bias toward those scenes that most closely match the conditions under which they smoke in the natural environment.

These applications of the dot-probe task to the study of smoking behavior remain to be explored. The present paper documents that the task is sensitive to differences in smoking history across groups. Current smokers in two studies showed significantly enhanced attention to smoking photographs compared with non-smokers. These results were obtained in the absence of explicit control over smoking behavior and across groups with large variations in overall reaction time performance. 
Furthermore, former smokers showed levels of bias intermediate in magnitude to the other two groups. These findings encourage further use of the dot-probe task as a measure of attentional bias in smokers.

\section{Acknowledgements}

These studies were supported by NIDA Center Grant \#P60-DA-05186 and by a VISN grant from the US Department of Veteran's Affairs.

\section{References}

Abrams, D.B., Monti, P.M., Carey, K.B., Pinto, R.P., Jacobus, S.I. 1988. Reactivity to smoking cues and relapse: two studies of discriminant validity. Behav. Res. Ther. 26, 225-233.

Baker, T.B., Morse, E., Sherman, J.E. 1987. The motivation to use drugs. A psychobiological analysis of urges. In: Rivers, C. (Ed.), The Nebraska Symposium on Motivation: Alcohol Use and Abuse. University of Nebraska Press, Lincoln, NE, pp. 257-323.

Cepeda-Benito, A., Tiffany, S.T. 1996. The use of a dual-task procedure for the assessment of cognitive effort associated with cigarette craving. Psychopharmacology 127, 155-163.

Childress, A.R., McLellan, A.T., O'Brien, C.P. 1986. Role of conditioning factors in the development of drug dependence. Psychiatr. Clin. North Am. 9, 413-425.

Droungas, A., Ehrman, R.N., Childress, A.R., O'Brien, C.P. 1995. Effect of smoking cues and cigarette availability on craving and smoking behavior. Addict. Behav. 20, 657-673.

Author, A. 1995. In: Drummond, D.C., Tiffany, S.T., Glautier, S., Remington, B. (Eds.), Addictive Behavior: Cue Exposure Theory and Practice. Wiley, New York.

Gotlib, I.H., Crane, D.B. 1987. Construct accessibility and clinical depression: a longitudinal investigation. J. Abnorm. Psychol. 96, 199-204.

Gross, T.M., Jarvik, M.E., Rosenblatt, M.R. 1993. Nicotine abstinence produces content-specific Stroop interference. Psychopharmacology 110, 333-336.

Johnson, B.H., Thayer, J.F., Laberg, J.C., Asbjornsen, A.E. 1997. Attentional bias in active smokers, abstinent smokers, and nonsmokers. Addict. Behav. 22, 813-817.

Lubman, D.I., Peters, L.A., Mogg, K., Bradley, B.P., Deakin, J.F.W 2000. Attentional bias for drug cues in opiate dependence. Psychol. Med. 30, 169-175.

MacLeod, C., Mathews, A. 1988. Anxiety and the allocation of attention to threat. Q. J. Exp. Psychol. 40, 653-670.

MacLeod, C., Mathews, A., Tata, P. 1986. Attentional bias in emotional disorders. J. Abnorm. Psychol. 95, 15-20.

Mathews, A.M, Mogg, K., Kentish, J., Eysenck, M. 1995. Effect of psychological treatment on cognitive bias in generalized anxiety disorder. Behav. Res. Ther. 33, 293-303.

Mattia, J.I., Heimberg, R.G., Hope, D.A. 1993. The revised Stroop color-naming task in social phobics. Behav. Res. Ther. 31, 305313.

McNair, D.M., Lorr, M., Droppleman, L.F., 1971. Profile of Mood States. Educational and Industrial Testing Service, San Diego, CA.

McNally, R.J., Kaspi, S.P., Riemann, B.C., Zeitlin, S.B. 1990. Selective processing of threat cues in posttraumatic stress disorder. J. Abnorm. Psychol. 99, 398-402.
Mogg, K., Mathews, A., Eysenck, M. 1992. Attentional bias to threat in clinical anxiety states. Cogn. Emot. 6, 149-159.

Mogg, K., Bradley, B.P., Hallowell, N. 1994. Attentional bias to threat: Role of trait anxiety, stressful events, and awareness. Q. J. Exp. Psychol. 47A, 841-864.

Mogg, K., Bradley, B.P., Williams, R. 1995. Attentional bias in anxiety and depression: the role of awareness. Br. J. Clin. Psychol. 34, 1736.

Mogg, K., Bradley, B.P., De Bono, J., Painter, M. 1997. Time course of attentional bias for threat information in non-clinical anxiety. Behav. Res. Ther. 35, 297-303.

Mogg, K., Bradley, B.P., Harpfreet, H., Lee, S. 1998. Selective attention to food-related stimuli in hunger: are attentional biases specific to emotional and psychopathological states, or are they also found in normal drive states? Behav. Res. Ther. 36, 227-237.

Niaura, R.S., Rohsenow, D.J., Binkoff, J.A., Monti, P.M., Pedraza, M., Abrams, D.B. 1988. Relevance of cue reactivity to understanding alcohol and smoking relapse. J. Abnorm. Psychol. 97, $133-152$.

Niaura, R.S., Abrams, D.B., Pedraza, M., Monti, P.M., Rohsenow, D.J. 1992. Smokers' reactions to interpersonal interaction cues and presentation of smoking cues. Addict. Behav. 17, 557-566.

O’Brien, C.P., Childress, A.R., Ehrman, R., Robbins, S.J. 1998. Conditioning factors in drug abuse: can they explain compulsion? J. Pharmacol. 12, 15-22.

Robbins, S.J., Ehrman, R.N. 1992. Designing studies of drug conditioning in humans. Psychopharmacology 106, 143-153.

Robinson, T.E., Berridge, K.C. 1993. The neural basis of drug craving: an incentive-sensitization theory of addiction. Brain Res. Rev. 18, $247-291$

Sayette, M.A., Hufford, M.R. 1994. Effects of cue exposure and deprivation on cognitive resources in smokers. J. Abnorm. Psychol. $103,812-818$.

Shiffman, S.M., Jarvik, M.E. 1976. Smoking withdrawal symptoms in two weeks of abstinence. Psychopharmacology 50, 35-39.

Siegel, S. 1979. The role of conditioning in drug tolerance and addiction. In: Keehn, J.D. (Ed.), Psychopathology in Animals: Treatment and Research Implications. Academic Press, New York, pp. $143-168$

Stewart, J., deWit, H., Eikelboom, R. 1984. The role of unconditioned and conditioned drug effects in the self-administration of opiates and stimulants. Psychol. Rev. 91, 251-268.

Tiffany, S.T. 1990. A cognitive model of drug urges and drug-use behavior: role of automatic and non-automatic processes. Psychol. Rev. 97, 147-168.

Townshend, J.M., Duka, T., 2001. Attentional bias associated with alcohol cues: differences between heavy and occasional social drinkers. Psychopharmacology, DOI 10.1007/s002130100764.

Waters, A.J., Feyerabend, C. 2000. Determinants and effects of attentional bias in smokers. Psychol. Addict. Behav. 14, 111-120.

Watts, F.N., McKenna, F.P., Sharrock, R., Trezise, L. 1986. Colour naming of phobia related words. Br. J. Psychol. 77, 97-108.

Wertz, J.M., Sayette, M.A. 2001. Effects of smoking opportunity on attentional bias in smokers. Psychol. Addict. Behav. 15, 268-271.

Wikler, A. 1965. Conditioning factors in opiate addiction and relapse. In: Wilner, D.I., Kassenbaum, G.G. (Eds.), Narcotics. McGrawHill, New York, pp. 85-100.

Williams, J.M.G., Mathews, A., MacLeod, C. 1996. The emotional Stroop task and psychopathology. Psychol. Bull. 120, 3-24.

Williams, J.M.G., Watts, F.N., MacLeod, C., Mathews, A. (Eds.),Cognitive Psychology and Emotional Disorders, second ed.. Wiley, New York 1997.. 